\title{
COMPARING ENTROPY MEASURE OF IDEA LINKS IN DESIGN PROTOCOLS
}

Linkography entropy measurement and analysis of different conditioned design sessions

JEFF WT KAN, ZAFER BILDA AND JOHN S GERO

University of Sydney, Australia

\begin{abstract}
This paper uses Shannon's entropy of information to measure linkographs of twelve design sessions which involved six architects in two separate design processes. In one they were not allowed to sketch (blindfolded condition), and in the other they were allowed to sketch. A previous study showed that the architects' overall cognitive activity in the blindfolded condition dropped below their activity in the sketching condition, approximately after 20 minutes (half way) during the timeline of the design sessions. This drop in performance was explained by higher cognitive demands and limitations of visuo-spatial working memory in the blindfolded conditions. Assuming that moves in a linkograph are the manifestation of ideas and entropy indicates the idea development potential, we tested whether entropy of idea links would also drop after half way during the blindfolded sessions. The results show that the visuospatial working memory load does not have negative effects on idea development. Further, we discuss that the idea development could be dependent more on a conceptual modality than the visuo-spatial modality.
\end{abstract}

\section{Background}

This section presents of the necessary background for this study; they are the construction of linkography, the measurement of linkography using Shannon's entropy, and the theory and method of blindfolded designing experiment.

\subsection{LINKOGRAPH}

Linkography was first introduced to protocol analysis by Goldschmidt (1990) to assess design productivity of designers. It is a technique used in 
protocol analysis to study designers. The design protocol is decomposed into small units called "design moves". Goldschmidt defines a design move as: "a step, an act, an operation, which transforms the design situation relative to the state in which it was prior to that move" (Goldschmidt 1995), or "an act of reasoning that presents a coherent proposition pertaining to an entity that is being designed" (Goldschmidt 1992). A linkograph is then constructed by linking related moves. It can be seen as a graphical network of associated moves that represents the design session. Figure 1 is a linkograph with 3 moves.

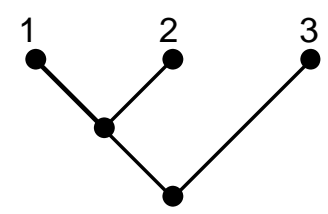

Figure 1. A linkograph with 3 moves, both moves 2 and 3 are related to move 1 but move 2 and move 3 are not related.

The design process can then be looked at in terms of the patterns of moves associations. Goldschmidt identified two types of links: backlinks and forelinks. Backlinks are links of moves that connect to previous moves, in Figure 1 moves 2 and 3 are backlinked to move 1 . Forelinks are links of moves that connect to subsequent moves, move 1 is forelinked to moves 2 and 3. Conceptually they are very different: "backlinks record the path that led to a move's generation, while forelinks bear evidence to its contribution to the production of further moves" (Goldschmidt 1995). Link index and critical moves were devised as indicators of design productivity. Link index is the ratio between the number of links and the number of moves, and critical moves are design moves that are rich in links, they can be forelinks, backlinks, or both. In her exposition, design productivity is positively related to the link index and critical moves, that is, a higher value of link index and critical moves indicates a more productive design process.

This link index and critical moves approach is biased towards highly linked linkograph because a saturated linkograph will have a high value of link index and critical moves. Kan and Gero (2005a) argue that a fully saturated linkograph indicates no diversification of ideas, hence less opportunity for quality outcomes.

\subsection{INFORMATION THEORY}

Following the above argument, empty linked and fully linked linkographs are not interesting, we speculate, intuitively, a randomly linked linkograph embodies a balanced process that embraces integration and diversification of ideas. This directs us to Shannon's (1948) concept of entropy as a measure 
of information. In Shannon's information theory, the amount of information carried by a message or symbol is based on the probability of its outcome. If there is only one possible outcome, then there is no additional information because the outcome is known. Information is transmitted through recognizable symbols predetermined by the source and receiver. Shannon demonstrated that the amount of information, entropy $(\mathrm{H})$, can be measured in terms of the probabilities of occurrence of the set of symbols.

$$
\mathrm{H}=\mathrm{p} 1 * \mathrm{~h}(\mathrm{p} 1)+\mathrm{p} 2 * \mathrm{~h}(\mathrm{p} 2)+\ldots+\mathrm{pN} * \mathrm{~h}(\mathrm{pN})
$$

Where $\mathrm{p} 1, \ldots \mathrm{pN}$ are probabilities corresponding to $\mathrm{N}$ states and $\mathrm{h}(\mathrm{p})$ is the information-generating function devised by Shannon which he derived to be $-\log b(p)$.

$$
\text { Therefore } \mathrm{H}=-\sum_{\mathrm{i}=1}^{\mathrm{n}} \mathrm{p}_{\mathrm{i}} \log _{\mathrm{b}}\left(\mathrm{p}_{\mathrm{i}}\right) \text { with } \sum_{\mathrm{i}=1}^{\mathrm{n}} \mathrm{p}_{\mathrm{i}}=1
$$

Section 2.3 will describe how this formula can be applied to calculate the entropy of a linkograph.

\subsection{SKETHCING AND BLIDNFOLDED DESIGNING}

Reviewing the literature in design studies and cognitive psychology, we can present two views on imagery and sketching activities in design, which also make a distinction between them. In the first view, sketching directly produces images and ideas externalized on paper and then the designer starts to have a dialogue with them via their perceptual mechanisms. In this way, the design problem space is explored, and restructured through this dialogue (Schon and Wiggins 1992; Goldschmidt 1991; Suwa Purcell and Gero 1998). In the second view, during the use of imagery alone for designing (designing without sketching), a designer has to accumulate considerable amount of knowledge/meaning before an image is generated, which suggests concept formation without drawings and thus without direct perceptual input. As with sketching activity, there is the dialogue with the images to restructure the design space; this probably is constrained within working memory capacities (Logie 1995).

Do architects necessarily start designing with external representations in the early stages of design? Anecdotal examples are often quoted of major architects such as Frank Lloyd Wright who could conceive of and develop a design entirely using imagery with an external representation of the design only being produced at the end of the process (Franklin 2003). Then it should be possible for some designers to develop and maintain an internal 
designing activity for a prolonged time. We refer to this activity as the use of imagery alone in designing.

Athavankar (1997) conducted an experiment where an industrial designer was required to design a product in his imagery (with an eye mask on), so that he had no access to sketching and the visual feedback it provides. The study showed that expert designers may be able to use imagery alone in the conceptual design phase, before externalizing their design thoughts. Similar results were obtained in a study with software designers (Petre and Blackwell 1999) where they were required to design using their mental imagery alone. The results of the study are qualitative, informing the possible cognitive processes and mechanisms that might be involved in blindfolded designing.

Bilda et al (2005) studied architects with an approach similar to Athavankar's (1997) study. At the end of the blindfolded designing, the participants were asked to quickly sketch the design solution they held in their minds. The solutions were assessed by judges and the results showed that the participants did reasonably well. Thus, when the participants were blindfolded, they were able to produce designs by using their cognitive resources to create and hold an internal representation of the design.

In another study, Bilda and Gero (2005) presented the cognitive activity differences of three expert architects when they design in blindfolded and sketching conditions. It was observed that all participants' overall cognitive activity in the blindfolded condition dropped below their activity in the sketching condition approximately after 20 minutes during the timeline of the design sessions. This drop in performance was explained by higher cognitive demands and limitations of visuo-spatial working memory in the blindfolded conditions. The results within the small group of participants showed that sketching off-loaded visuo-spatial working memory.

In this paper we question whether the working memory limitations have an impact on idea development. Evidence in working memory research supports that the cognitive load should be higher in a blindfolded condition since image maintenance and synthesis of images requires more executive control resources (Pearson et al 1999, Baddeley et al 1998). Other empirical studies on visuo-spatial working memory (VSWM) also show that the capacity of the VSWM is limited when visuo-spatial tasks are done using imagery (Ballard et al 1995, Walker et al 1993, Phillips and Christie 1997). Similar results have been obtained for phonological loop of the working memory, when verbal tasks were performed using imagery (Baddeley 1986). We presume that the design ideas could have a visuo-spatial mode and a verbal mode in imagery working in parallel. Since it was shown that there exists a working memory limitation during use of imagery, the idea development could slow down after a while during the timeline of the 
blindfolded designing activity. On the other hand sketching activity could support and improve the idea development activity, since working memory is off-loaded continuously.

\section{Method}

The six architects who participated in the study (2 female and 4 male) have each been practicing for more than 15 years. Architects A1 and A2 run their own companies and have been awarded prizes for their designs in Australia; Architect A3 is a senior designer in a well-known architectural firm. The three participants were teaching part-time in design studios. A4 works for one the Australia's largest architectural company and he has been the leader of many residential building projects from small to large scales. A5 is one of the founders and director of an award wining architectural company. A6 is a very famous residential architect in Sydney, and he directs his company known by his name with 50 employees.

\subsection{DESIGN OF THE EXPERIMENTS}

We conducted think-aloud designing experiments with the six architects. The first group of the three architects, A1, A2 and A3 were initially engaged in a design process where they are not allowed to sketch. This phase is called the experiment condition where they received design brief 01 . One month after the experiment condition the same three architects were engaged in a design process where they are allowed to sketch. This phase is called the control condition where they receive design brief 02 . Design brief 01 requires designing a house for two artists: a painter and a dancer. The house is to have two studios, an observatory, a sculpture garden and living, eating, sleeping areas. Design brief 02 requires designing a house on the same site as design brief 01 but this time for a couple with 5 children aged from 3 to 17, that would accommodate children and parent sleeping areas, family space, study, guest house, eating and outdoor playing spaces.

The second group of the three architects was first engaged in the sketching (control condition) session, where they received design brief 02 . Then after one month they were under the experiment condition working on design brief 01 , where they were not allowed to sketch.

The set-up of the study for both experiment and control conditions has a digital video recorder with a lapel microphone, directed at the designer. In the experiment condition, the architects were required to put on a blindfold and start thinking aloud, Figure 2(a). At the end of the 45 minute session they were asked to take off the blindfold and quickly sketch what they held in their minds in the allowed 5 minute period. They were instructed that changes were not permitted to the design solution they originally had in their minds. The details of the experimental procedure can be found in Bilda et al 
(2005). During the 45 minute period of the sketching condition, the architects were required to think aloud and sketch at the same time.

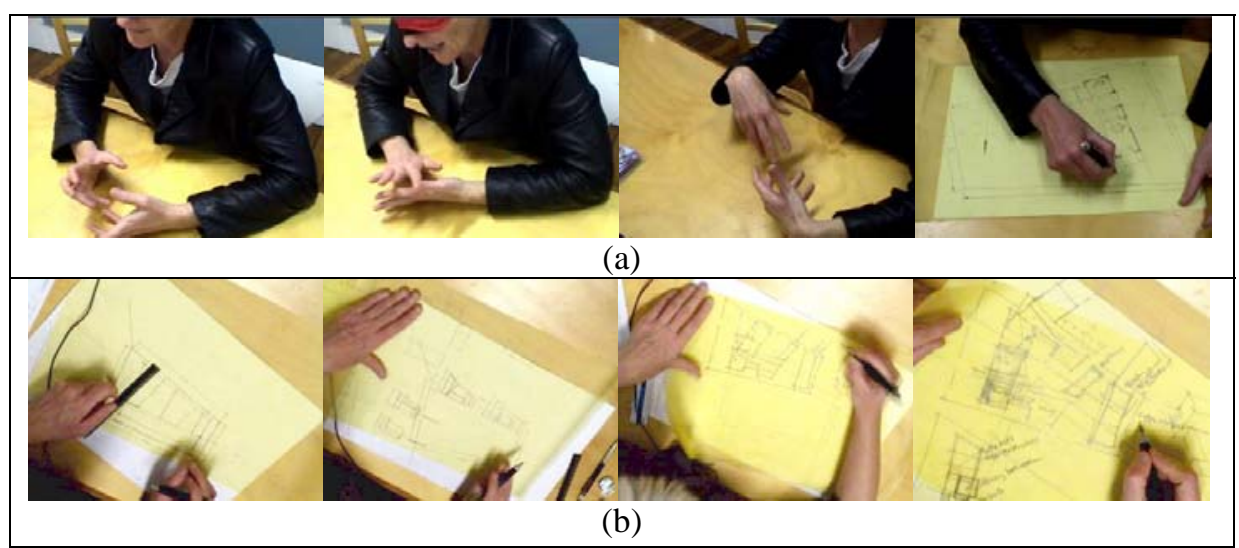

Figure 2. (a) blindfolded session followed by quick sketching, (b) sketching session

\subsection{CODING THE LINKS}

The linkography technique involves dividing the protocol into design moves (Goldschmidt 1991) and looking at the design process in terms of relationships created by the links between those moves. In this study we segmented the protocols according to designers' intentions (Suwa et al 1998). Suwa et al. (1998) discussed that this segmentation technique was similar to the notion of design moves, thus we used the same segmented intervals in the process of coding links.

The process of connecting the ideas between the segments is carried out in two runs. In the first run, the coder starts from the first segment and sequentially connects the ideas, and revisited meanings between the segments. The analyzer relies on the verbalization only while linking the ideas in the blindfolded designing protocols. During linking the ideas of sketching protocols, video footage for each segment was visited as well. Linking the ideas in protocols could be difficult task, if the analyzer loses track of the ideas developed previously along the timeline of the protocol session. In order to prevent the missing links and for linking the ideas more reliably, we employed a technique that involves a word search in order to detect the words used more frequently such that the analyzer ends up with a list of frequently repeated words in each segment. The next stage is browsing through the selected segments, to confirm that the words are used in the appropriate context. If the ideas are related, then these segments are connected to each other. This procedure helps us to connect the meanings, which are distant from each other and which might have been missed in a sequential analysis. 


\subsection{ENTROPY MEASUREMENT}

In our measure of linkograph entropy we are interested not only in the number of linked moves but also the distribution of those links. Two extremes example are: an empty linked (none of the moves is related) and a fully linked (all the moves are linked) linkograph. An empty linked linkograph can be considered as a non-converging process with no coherent ideas and a fully linked linkograph stands for a total integrated process with no diversification. In both cases the opportunities for idea development is very low. This line of reasoning can be expressed in terms of entropy; if we randomly pick a move in an empty linked linkograph we can be sure that it has no links. This sounds obvious but if we consider this linkograph as a carrier with zero information content, because the outcome is known, it will have zero entropy. Similarly, a fully linked linkograph will also have zero entropy.

In order for entropy measurement of linkograph to be meaningful, we follow Kan and Gero's (2005b) measurement method, which based on the conceptual difference of forelink, backlink, and horizontal link (called "horizonlink"). Entropy is measured in rows of forelinks, backlinks, and horizonlinks separately, Figures 3 (a), (b), and (c).

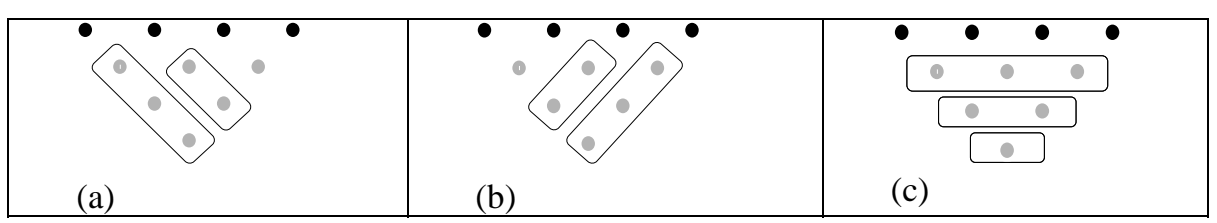

Figure3. (a) Measuring entropy of forelinks of each row, (b) measuring entropy of backlinks of each row, and (c) measuring entropy of horizonlinks.

Entropy is calculated according to the probability of ON/OFF of each link. Following Shannon's theory, formula (2), $\mathrm{H}$ becomes:

$$
-\mathrm{p}(\mathrm{ON}) \log (\mathrm{p}(\mathrm{ON}))-\mathrm{p}(\mathrm{OFF}) \log (\mathrm{p}(\mathrm{OFF}))
$$

Each row will receive an entropy value between 0 and 1 . A higher value signifies a good mix of ON and OFF links. A more detailed analysis of formula 3 together with examples of the calculations is described in Kan and Gero (2005b). They concluded that forelink entropy measures the idea generation opportunities in terms of new creations or initiations. Backlink entropy measures the opportunities according to enhancements or responses. Horizonlink entropy measures the opportunities relating to cohesiveness and incubation (Kan and Gero 2005b). 


\section{Results}

A previous study, using the results from the first group of architects, showed that the overall cognitive activity in the blindfolded condition dropped below the activity in the sketching condition approximately after 20 minutes (half way) of the design sessions (Bilda and Gero 2005). This drop in performance can be explained by higher cognitive demands and limitations of visuo-spatial working memory (VSWM) in blindfolded conditions. However, with this drop of cognitive activity, in the blindfolded condition the architects can still produce satisfactory designs. Their design outcomes, sketches, were judged by a qualified jury of three designers. Surprisingly, all the blindfolded sessions received a higher score as compared to the corresponding sketch sessions. We attempt to explore these phenomena by using entropy measures of the linkograph.

\subsection{LINKOGRAPHS OF THE SESSIONS}

After coding the links, 12 linkographs of the sessions had been produced; they exhibit different patterns that reflect different design processes. For example the linkograph in Figure 4(a) reflects a relatively holistic design process (the linkograph is well integrated) while the linkograph in Figure 4 (b) represents a process of trying out different options (there are obvious clusters in the linkograph). This observation was confirmed with our qualitative analysis.

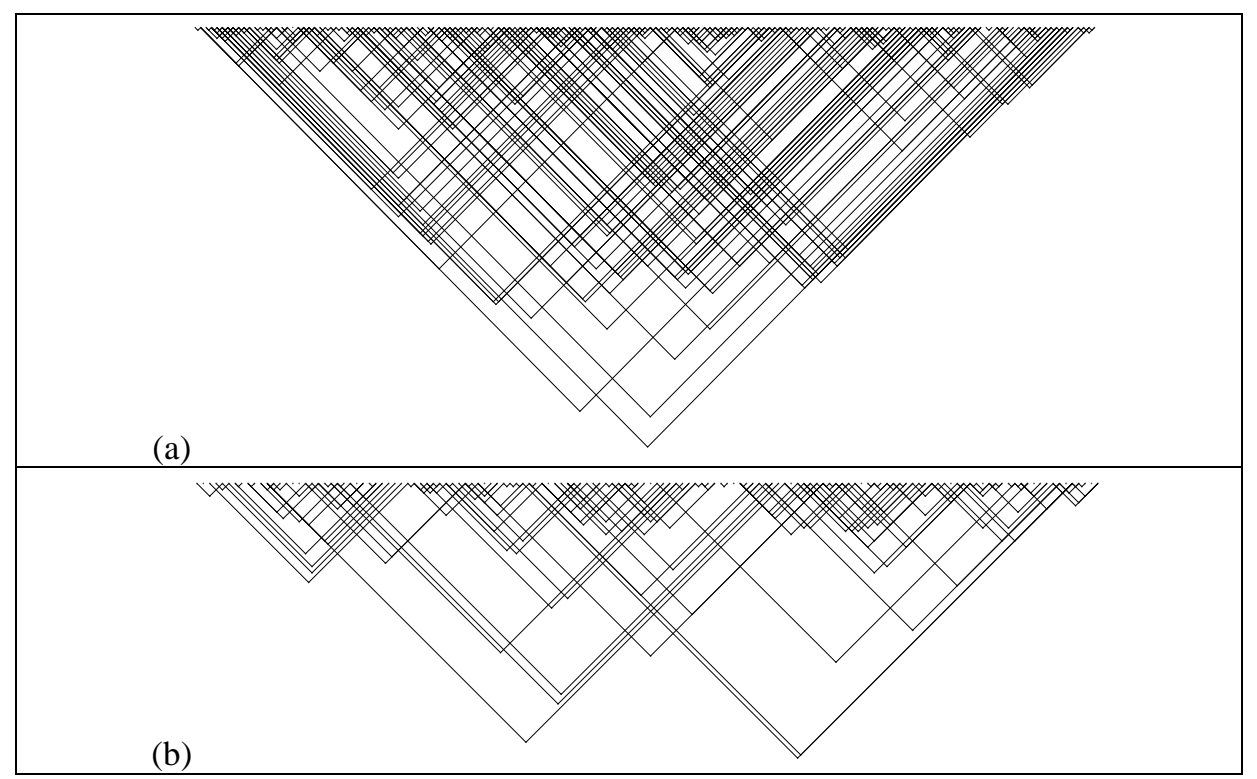

Figure 4. (a) Linkograph of a sketch session, (b) Linkograph of a blindfolded session 
The detailed qualitative analysis of the 12 linkographs is beyond the scope of this paper. Here we examine whether the drop of cognitive activity in the blindfolded session is reflected in the linkograph. Looking at the linkograph, some of the blindfolded sessions seem to suggest a more productive process according to Goldschmidt's definition due to the visual density of links. However, we cannot track down where the cognitive activity started to drop. Then we count the moves of the first 20 minutes in all the sessions as compared to the rest of the session, Table 1 . In general the blindfolded sessions do have slightly more moves in the first half of the session, this is consistence with the previous result as cognitive activity is reflected in moves, but visually the number of links does not seem to drop in the second half.

TABLE 1. Number of moves in the first 20 minutes and the rest of the session

\begin{tabular}{|l|l|l|l|l|l|l|}
\hline \multirow{2}{*}{} & \multicolumn{3}{|c|}{ Blindfolded No. of Moves } & \multicolumn{3}{c|}{ Sketch No. of Moves } \\
\cline { 2 - 7 } & 20 min & Rest & Total & 20 min & Rest & Total \\
\hline Architect 1 & 89 & 78 & 167 & 68 & 77 & 145 \\
\hline Architect 2 & 63 & 91 & 154 & 77 & 107 & 184 \\
\hline Architect 3 & 87 & 82 & 169 & 65 & 77 & 142 \\
\hline Architect 4 & 92 & 75 & 167 & 74 & 95 & 169 \\
\hline Architect 5 & 73 & 72 & 145 & 91 & 62 & 153 \\
\hline Architect 6 & 69 & 53 & 122 & 71 & 101 & 172 \\
\hline Average & $\mathbf{7 8 . 8 3}$ & $\mathbf{7 5 . 1 7}$ & $\mathbf{1 5 4 . 0 0}$ & $\mathbf{7 4 . 3 3}$ & $\mathbf{8 6 . 5 0}$ & $\mathbf{1 6 0 . 8 3}$ \\
\hline
\end{tabular}

\subsection{ENTROPY OF THE SESSIONS}

We use formula (3) to calculate the forelink and backlink entropy of each move in a linkograph. In order to get a picture of the whole session, we add the entropy of each move to get the total forelink and backlink entropy of a session. Likewise, we get the total horozonlink entropy by adding the entropy of all the horizontal rows. For the ease of comparison, we divide the entropy by the total number of moves in that session. Table 2 shows the normalized results of entropy of each session together with their link index. Average values are taken in accordance to the two groups of experiments. Overall the blindfolded sessions have higher entropy and link index in both groups. Investigating individual architect, only the first architect in the first group does not follow this trend. 
TABLE 2. Entropy and link index of each session

\begin{tabular}{|c|c|c|c|c|c|c|}
\hline & & $\begin{array}{l}\mathrm{BH} / \\
\text { move } \\
\mathrm{s}\end{array}$ & $\begin{array}{l}\text { FH/ } \\
\text { move } \\
\text { s }\end{array}$ & $\begin{array}{l}\mathrm{HH} / \\
\text { moves }\end{array}$ & Total & $\begin{array}{l}\text { Link } \\
\text { Index }\end{array}$ \\
\hline \multirow{8}{*}{ 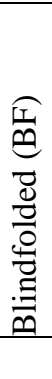 } & BF 1 & 0.125 & 0.122 & 0.060 & 0.307 & 1.20 \\
\hline & BF 2 & 0.161 & 0.155 & 0.066 & 0.383 & 1.68 \\
\hline & BF 3 & 0.143 & 0.140 & 0.055 & 0.338 & 1.28 \\
\hline & $a v$ & 0.143 & 0.139 & 0.060 & 0.343 & 1.39 \\
\hline & BF 4 & 0.240 & 0.220 & 0.093 & 0.553 & 2.48 \\
\hline & BF 5 & 0.224 & 0.193 & 0.082 & 0.499 & 2.18 \\
\hline & BF 6 & 0.188 & 0.189 & 0.105 & 0.481 & 2.50 \\
\hline & $a v$ & 0.217 & 0.201 & 0.093 & 0.511 & 2.39 \\
\hline \multirow{8}{*}{ 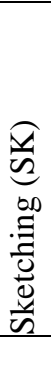 } & SK 1 & 0.137 & 0.124 & 0.077 & 0.337 & 1.41 \\
\hline & SK 2 & 0.157 & 0.150 & 0.065 & 0.373 & 1.48 \\
\hline & SK 3 & 0.124 & 0.131 & 0.044 & 0.299 & 1.20 \\
\hline & $a v$ & 0.139 & 0.135 & 0.062 & 0.336 & 1.36 \\
\hline & SK 4 & 0.227 & 0.203 & 0.098 & 0.529 & 2.41 \\
\hline & SK 5 & 0.176 & 0.125 & 0.071 & 0.372 & 1.68 \\
\hline & SK 6 & 0.184 & 0.175 & 0.063 & 0.422 & 1.76 \\
\hline & $a v$ & 0.196 & 0.168 & 0.077 & 0.441 & 1.95 \\
\hline \multicolumn{7}{|c|}{ BH: entropy of back links } \\
\hline
\end{tabular}

Table 2 shows that first group of three architects' blindfolded (BF) and sketch (SK) conditions have very close link indices $(1.39,1.36)$ and entropy values $(0.343,0.336)$. For the second group of architects, the link index and total entropy values are higher in the BF condition compared to their SK condition (0.511, 0.441 and 2.39, 1.95). We observed that for the second group of architects the link index and the entropy of the blindfold sessions are the highest. This difference could be due to the experimental condition where the second group received the sketching exercise first and the blindfolded exercise later. The second group had an increased familiarity with the problem space, including the site geometry and the environmental factors around the building. This familiarity with the problem space could have improved the second group potential for idea development.

In general backlink entropy is higher than the forelink entropy, with the exception of one sketch condition and one blindfold condition. We assume that forelink entropy indicates the idea generate potential based on new initiation which may reflect creativity. The architect who had the forelink entropy higher than the backlink entropy in the sketch session received the 
highest rank by the judges in the categories of "Innovative" and "Creative". The relationship between forelink entropy and creativity needs further investigation and is inconclusive at this moment.

The horizonlink entropy is much lower than forelink and backlink entropies due to the fundamental dissimilarity. Forelink and backlink entropy examine linkages of each move, but horizonlink examines the cohesiveness of the moves. This measure is more meaningful when in a team designing environment (Akaike 1973).

\subsection{CHANGE OF ENTROPY OVER TIME}

As we observe the linkographs of Figures 4, it is easy to infer that the entropy varies across the time line. There are two approaches for monitoring these changes, one uses a fixed time frame as a reference window and the other a fixed number of moves as the width of window. In this calculation we ignore those linked nodes outside this reference window. Mathematically, it is easier to use fixed number of moves as reference as the procedure can be automated. Figure 5 shows A1's backlink, forelink and horizontal link entropy changes, using 28 moves as a window width.

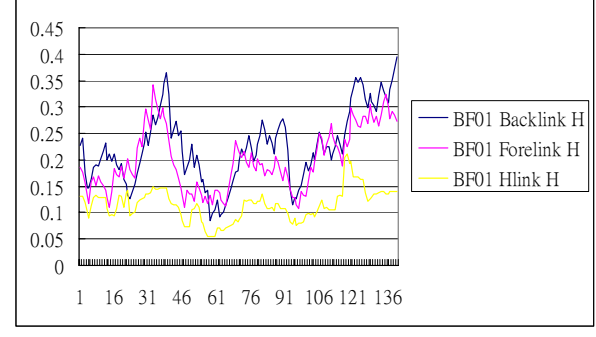

(a)

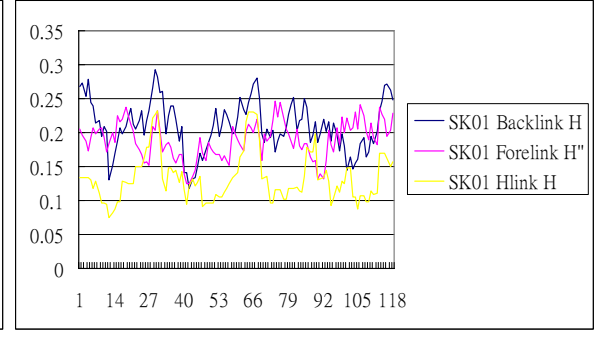

(b)

Figure 5. Change of entropy (a) in BF condition (b)in SK condition

We can identify two regularities in these graphs: the relationship between the three types of link entropies; and the overall trend. When we look at the relationships between the backlinks, forelinks and horizontal links, they show similar relationships but with different shapes for all architects and conditions. We have not studied the implication of these trends yet.

In order to compare the entropy graphs in the two conditions with reference to time, we have to assume the moves are of similar duration and we scale up the backlink H graph of Figure 5(b) to match it with the one in Figure 5(a), Figure 6. 


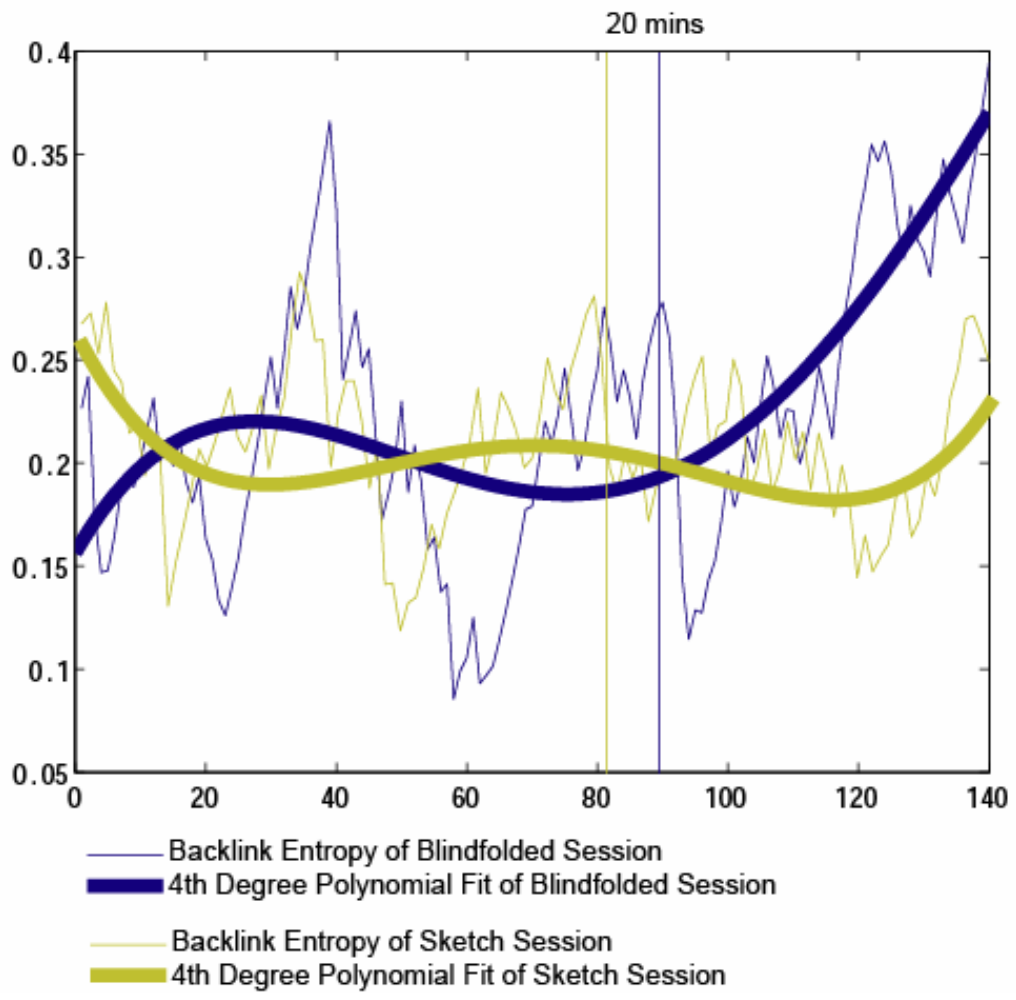

Figure 6. Comparing backlink entropy of the same architect in the two different condition

The moves at the 20 minutes mark for the blindfolded and sketch condition are 89 and 68 respectively. After scaling moves in the sketch condition the 20 minutes mark for the sketch session falls around 81 which is not too far for our qualitative analysis. As we can see the graph in the blindfolded condition drops very quickly from move 90 to 94 but starts climbing until the end, this graph remains above the sketch condition after move 101.

\subsection{HYPOTHESIS TESTING}

In this section we would like to test if the entropy of idea links drops half way through the blindfolded design process, due to working memory limitations. We assume idea development is positively co-related to entropy. We use the move at 20 minutes to divide the linkograph, Figure 7, and calculate the entropy of the first and second half of the linkographs to compare if there is a significant different between the BF and SK conditions. We chose 20 minutes as a demarcation, based on a previous study, where the 
first three participants' cognitive performance in BF conditions dropped below their performance in SK conditions, after around 20 minutes (Bilda and Gero 2005).

In this calculation we ignore those linked nodes outside the shaded triangles. The results in Table 3 show that in both conditions there is a drop in entropy in the second half of the sessions. In the sketch condition five out of the six has lower entropy in the second half; conversely half of blindfolded sessions have higher entropy.

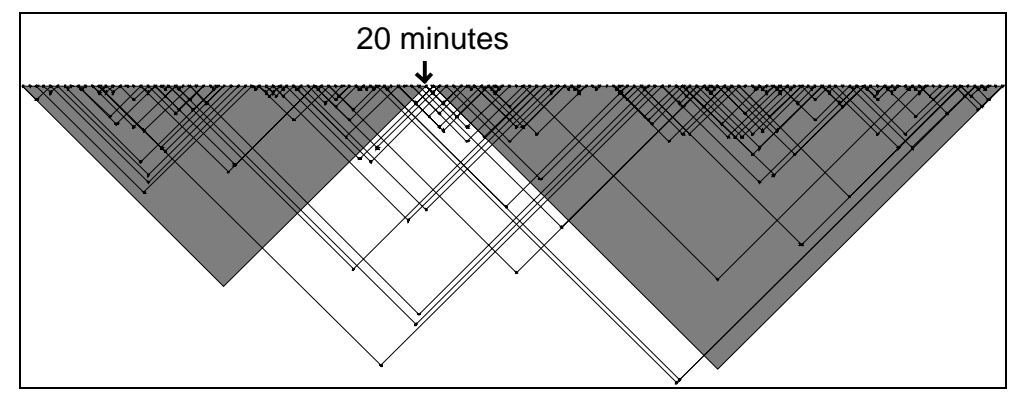

Figure 7. Calculating the entropies of the first and second half of the linkograph; only links within the gray triangle are counted

TABLE 3. Total normalized entropies of each Session

\begin{tabular}{|l|r|r|r|r|}
\hline & \multicolumn{2}{|c|}{ Blindfolded Entropies } & \multicolumn{2}{c|}{ Sketch Entropies } \\
\hline & First 20 min & \multicolumn{1}{|c|}{ The rest } & First 20 min & The rest \\
\hline Architect 1 & 0.343 & 0.411 & 0.450 & 0.398 \\
\hline Architect 2 & 0.650 & 0.498 & 0.484 & 0.448 \\
\hline Architect 3 & 0.406 & 0.441 & 0.461 & 0.518 \\
\hline Architect 4 & 0.743 & 0.670 & 0.783 & 0.588 \\
\hline Architect 5 & 0.911 & 0.679 & 0.603 & 0.529 \\
\hline Architect 6 & 0.857 & 0.875 & 0.652 & 0.540 \\
\hline Average & $\mathbf{0 . 6 5 2}$ & $\mathbf{0 . 5 9 6}$ & $\mathbf{0 . 5 7 2}$ & $\mathbf{0 . 5 0 3}$ \\
\hline
\end{tabular}

Generally, the entropy of the second half of the session is lower than the first half. This parallels our assumption that entropy measures the opportunities for idea generation. In the second half, designers are approaching the end of a session and the design converges to a particular approach, therefore fewer opportunities occur for idea development. Consequently the entropy values would be lower in the second half compared to the first. In order to understand what is happening to the idea 
links we have to refer back to formula (3) which Kan and Gero (2005b) plotted as shown in Figure 8.

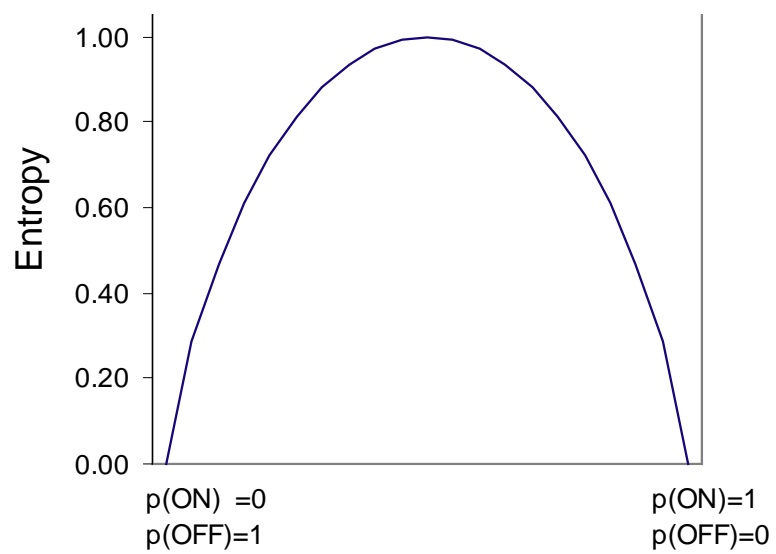

Figure 8. Maximum entropy when $\mathrm{p}(\mathrm{ON})=\mathrm{p}(\mathrm{OFF})=0.5$

This graph shows that when $p(\mathrm{ON})$ is between $\{0.35,0.65\}$, entropy $\mathrm{H}$ is over 0.93 that is if the links in a row are between $35 \%$ and $65 \%$ it will produce a very positive value. If the links are less then $5 \%$ or over $95 \%$, it will produce a very low $\mathrm{H}$ value (below 0.29 ). Either heavily linked or rarely linked second half linkograph will have low entropy values. Reviewing the 12 linkographs, we could conclude they all fit the second case; that is linked ideas are sparse. So a higher value of entropy implies more linkage among moves, hence better development of ideas. The results in Table 3 suggest that in the blindfolded condition, working with limited VSWM, there is no negative consequence on the inter-connectivity of ideas. This is confirmed by two tailed paired t-test with the null hypothesis being: there is a drop of entropy after 20 minutes in the blindfolded sessions. To test against the hypothesis we compute the entropy difference of the first 20 minutes and the rest of the session, Table 4. Then we paired the two sets of data according to the architect.

TABLE 4. Entropy differences

\begin{tabular}{|l|r|r|r|r|r|r|}
\hline & \multicolumn{1}{|c|}{ Pair 1 } & \multicolumn{1}{|c|}{ Pair 2 } & \multicolumn{1}{|c|}{ Pair 3 } & \multicolumn{1}{|c|}{ Pair 4 } & \multicolumn{1}{|c|}{ Pair 5 } & \multicolumn{1}{|c|}{ Pair 6 } \\
\hline BF diff & -0.068 & 0.152 & -0.036 & 0.072 & 0.232 & -0.017 \\
\hline SK diff & 0.053 & 0.036 & -0.057 & 0.195 & 0.074 & 0.112 \\
\hline
\end{tabular}

BF: Blindfolded condition

SK: Sketch condition

diff: entropy of the first 20 min - entropy of the rest

The t-test resulted is 0.82 , so the hypothesis is not supported for $\mathrm{p}=<$ 0.05. The development of linked idea does not drop half way through the 
blindfolded design process. The implication of this result is profound, contrary to usual beliefs; when VSWM is overloaded it may not have negative effects on idea development.

\section{Discussions and Conclusions}

Kan and Gero (2005b) showed that an entropy measure could be a useful tool to measure idea development, because it measures the chances of linking ideas to previous ones (backlinks) and chances of developing ideas that would be sought later (forelinks) during the design process. We interpreted this linkograph entropy measure as "the potential for idea development”. Then we tested whether working memory limitations has an impact on the potential for idea development.

The results showed that the idea development did not necessarily slow down after some time during the use of imagery alone. In our previous findings, perceptual activity for the three architects significantly slowed down after 20 minutes during the BF designing sessions. It was improved during sketching (Bilda and Gero, 2005). Perceptual activity, being related to the tasks in visuo-spatial working memory (VSWM), slowed down because of VSWM limitations. Perhaps, idea development is not dependent on a visuo-spatial modality, since idea development potential seemed not to be effected by working memory load.

In Bilda and Gero (2005) we also observed that the variances in functional activity (attaching meaning and purpose to things) were similar in BF and SK conditions. This implied that sketching did not add to (improve) production of meaning, however it improved perceptual activity. This result also suggested that the cognitive load (working memory load) might be related to perceptual activity rather than functional activity. The visuospatial tasks which require executive resources in working memory might create the cognitive load, but not necessarily the concept/meaning formation. In the same parallel assuming that idea development in imagery is conceptrelated rather than figure-related, results in this paper supports our previous findings.

We conclude that that the VSWM load does not have negative effects on idea development. Further, the idea development could be dependent more on a conceptual modality than the visuo-spatial modality.

\section{Acknowledgements}

This research supported by an International Postgraduate Research Scholarship and a University of Sydney International Postgraduate Award, facilities are provided by the Key Centre of Design Computing and Cognition. We would like to thank to the architects who participated in this study. 


\section{References}

Akaike, H: 1973, Information theory as an extension of the maximum likelihood principle, Second International Symposium on Information Theory, B Petrov and C Csaki, Akademiai Kiado, Budapest: 267-281.

Athavankar, UA: 1997, Mental imagery as a design tool, Cybernetics and Systems 28: 25-47.

Baddeley, AD: 1986, Working Memory, Oxford, Clarendon Press.

Baddeley, AD, Emsile, H, Kolodny, J and Duncan, J: 1998, Random generation and executive control of working memory, Quarterly Journal of Experimental Psychology 51A: 819-852

Ballard, DH, Hayhoe, MM, and Pelz, JB: 1995, Memory representations in natural tasks, Journal of Cognitive Neuroscience 7: 66-80.

Bilda, Z , Gero, JS and Purcell AT: 2005, To sketch or not to sketch: That is the question, Design Studies (to appear).

Bilda, Z and Gero, JS: 2005, Does sketching off-load visuo-spatial working memory? In Gero, JS and Bonnardel, N (eds) Studying Designers'05, Key Centre of Design Computing and Cognition, University of Sydney.

Franklin, T: 2003, Falling water rising: Frank Lloyd Wright, E.J. Kaufmann, and America's most extraordinary house, New York, Knopf.

Goldschmidt, G: 1990, Linkography: assessing design productivity, Cyberbetics and System '90, R Trappl. World Scientific, Singapore: 291-298.

Goldschmidt, G: 1992, Criteria for design evaluation: a process-oriented paradigm, Evaluating and Predicting Design Performance, YE Kalay. New York, John Wiley \& Son, Inc.: 67-79.

Goldschmidt, G: 1995, The designer as a team of one, Design Issue 16(2): 189-209.

Kan, JW and Gero, JS: 2005a, Acquiring information from linkography in protocol studies of designing, Design Studies (to appear).

Kan, WT and Gero, JS: 2005b, Can entropy indicate the richness of idea generation in team designing? CAADRIA05, New Delhi, India.

Logie, RH: 1995, Visuo-Spatial Working Memory, Hillsdale, Lawrence Erlbaum Associates, Publishers.

Pearson, DG Logie, RH and Gilhooly, KJ: 1999, Verbal representations and spatial manipulations during mental synthesis, European Journal of Cognitive Psychology 11 (3): 295-314

Phillips, WA and Christie, DFM: 1997, Components of visual memory, Quarterly Journal of Experimental Psychology 29: 117-133.

Schon, D A and Wiggins G: 1992, Kinds of seeing and their functions in designing, Design Studies 13(2): 135-156.

Shannon, CE: 1948, A mathematical theory of communication, The Bell System Technical Journal 27: 397-423.

Suwa, M, Purcell, T and Gero, JS: 1998, Macroscopic analysis of design processes based on a scheme for coding designers' cognitive actions, Design Studies 19 (4): 455-483.

Walker, P, Hitch, G, and Duroe A: 1993, The effect of visual similarity on short-term memory for spatial location: Implications for the capacity of visual short term memory, Acta Psychologica 83: 203-224. 